\title{
Active Power Filter Shape Class Model Predictive Controller tuning by Multiobjective Optimization
}

\author{
Carlos Cateriano Yáñez ${ }^{1}$, Jörg Richter², Georg Pangalos², Gerwald Lichtenberg ${ }^{3}$, Javier Sanchís \\ $\mathrm{Saez}^{4}$ \\ ${ }^{1}$ Universitat Politécnica de Valencia, Spain; ${ }^{2}$ Fraunhofer Institute for Silicon Technology, Hamburg \\ University of Applied Sciences, Germany, ${ }^{3}$ Hamburg University of Applied Sciences, Germany, \\ ${ }^{4}$ Department of Systems Engineering and Control, Universitat Politécnica de Valencia, Spain.
}

\begin{abstract}
In order to compensate the power quality issues that arise in distribution grids with high penetration of renewable energy sources, an active power filter device controlled by a novel model-based predictive controller, i.e. the linear state signal shaping model predictive controller, is implemented. This paper proposes the use of a Multiobjective Optimization evolutionary algorithm, i.e. the Multiobjective Differential Evolution with Spherical Pruning X, for the tuning of this novel controller. An application example for power quality compensation of a grid modeled as a switched system with four modes is given. The model includes nonlinear loads that introduce harmonic distortion and multiple consumer loads that enable the existence of conflicting objectives, typical of multiobjective optimization problems. A decision making strategy is developed in order to find the best controller parameters in a reasonable amount of time that enable the provision of optimal power quality services by balancing multiple objectives that can conflict with each other.
\end{abstract}

Keywords: Power quality, harmonic mitigation, load compensation, active power filters, model predictive control, multiobjective optimization. 


\section{Introduction}

As the share of renewable energy sources (RES) in distribution grids increases, several power quality challenges arise. Due to its intermittent nature, RES lead to voltage and frequency fluctuations in the grid that affect power quality. Moreover, as RES are connected via power converters, there is also a higher harmonic distortion pollution introduced by the switching power electronics involved (Liang, 2017).

A proven solution is the implementation of Active Power Filters (APF), which can compensate the unbalanced, harmonic, and reactive components of a load. In this context, the selection of an appropriate control strategy is critical (Kumar \& Mishra, 2016).

This paper uses a novel Linear State Signal Shaping Model Predictive Control (LS ${ }^{3}$ MPC) approach (Cateriano Yáñez et al., 2018). This strategy forces the states of a linear system to follow specific linear dynamics, i.e. a shape class, by reformulating a quadratic Model Predictive Control (MPC). The LS $^{3}$ MPC can compensate harmonic distortion under different load scenarios, compared to classical APF control methods (Weihe et al., 2018).

The evolutionary algorithm Multiobjective Differential Evolution with Spherical Pruning X (spMODEx) (Reynoso Meza et al., 2017), is proposed for the tuning of the LS ${ }^{3}$ MPC control parameters, to enhance its power quality compensation capabilities. A simulation setup with a grid modeled as a switched system with four modes is developed as an application example. The guidelines for a decision making strategy process to choose the optimal controller parameters set are presented.

The paper is organized as follows. Section 2 introduces the models of the grid for the plant simulation and controller. In section 3, an overview of the harmonic shape class LS ${ }^{3} \mathrm{MPC}$ is given. Section 4 introduces the multiobjective optimization (MOO) setup, whereas section 5 , covers the experiment setup and the analysis of the simulation results. Finally, section 6 summarizes the findings and draws conclusions.

\section{Grid model with an active power filter}

This section introduces a general definition for state space models, followed by a description of the models used for the plant simulation and for the controller.

\subsection{State space models}

Linear systems dynamics can be represented by a discrete-time state space model

$$
\mathbf{x}(k+1)=\mathbf{A x}(k)+\mathbf{B u}(k)+\mathbf{E d}(k),
$$

with sampling time $t_{s}$ at $t=k t_{s}$, state vector $\mathbf{x} \in \mathbb{R}^{n}$, input vector $\mathbf{u} \in \mathbb{R}^{m}$, disturbance vector $\mathbf{d} \in \mathbb{R}^{z}$, system matrix $\mathbf{A} \in \mathbb{R}^{n \times n}$, input matrix $\mathbf{B} \in \mathbb{R}^{n \times m}$, and disturbance matrix $\mathbf{E} \in \mathbb{R}^{n \times z}$. 


\subsection{Plant model description}

A four mode switched grid circuit is given in Figure 1. From left to right, the supply voltage $V_{S}$ denotes the external grid coupling, followed by the transmission line TL1 into the point of common coupling PCC1. From PCC1, there is a feeder line F1 that leads to the rectifier D1 (shown connected) and a parallel load line L1. There is a second transmission line TL2 connecting PCC1 with PCC2. From PCC2, there is a feeder line F2 that leads to the rectifier D2 (shown disconnected), a parallel load line L2, and a compensation source CS. The switching of the rectifiers D1 and D2 introduce harmonic distortion to the system, which is to be compensated by the compensation source CS.

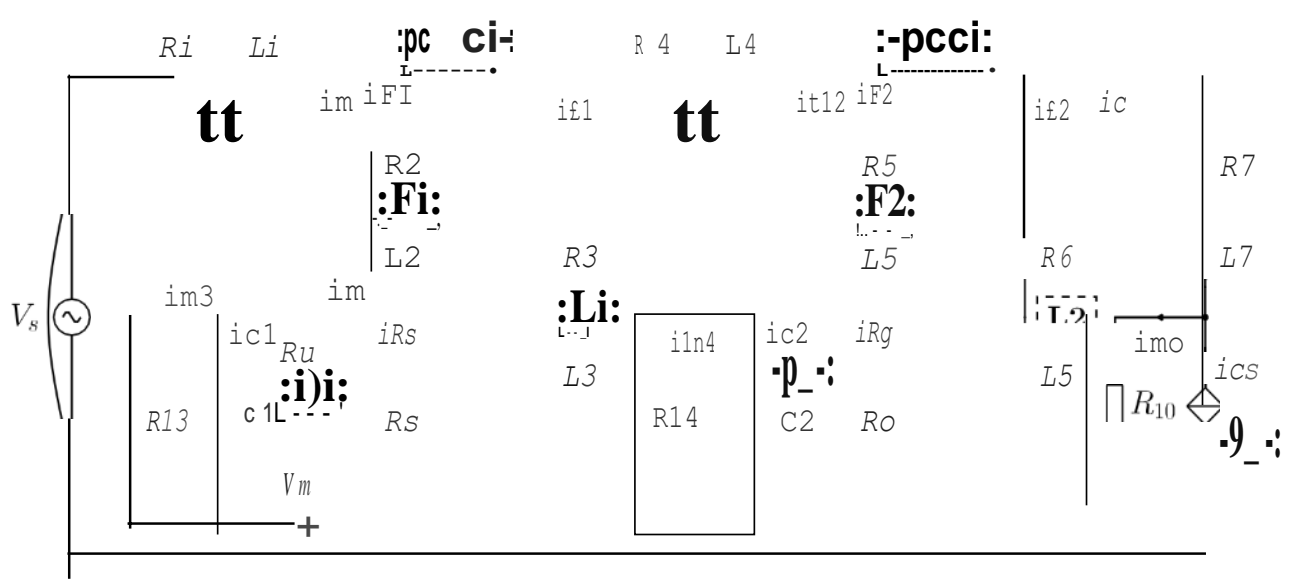

Figure 1. Grid circuit with rectifier D1connected and rectifier D2 disconnected (Richter, 2018).

Depending on the switching state (on/off) of the rectifiers D1 and D2, the system is in one of four mutually exclusive modes. The switching conditions are in Table 1. Each configuration gives a different state space model, which is discretized by zero-order hold, leading to the signal vectors

$$
\begin{aligned}
\mathbf{x} & =\left[\begin{array}{lllllllll}
i_{t l 1} & i_{t l 2} & i_{L 1} & i_{L 2} & i_{F 1} & i_{F 2} & i_{C} & V_{C 1} & V_{C 2}
\end{array}\right]^{\mathrm{T}}, \\
u & =i_{C S}, \\
\mathbf{d} & =\left[\begin{array}{lllll}
V_{s} & V_{D 1} & V_{D 2}
\end{array}\right]^{\mathrm{T}},
\end{aligned}
$$

with the corresponding matrices $\mathbf{A} \in \mathbb{R}^{9 \times 9}, \mathbf{B} \in \mathbb{R}^{9 \times 1}$, and $\mathbf{E} \in \mathbb{R}^{9 \times 3}$.

Table 1. Four mode switching conditions for rectifiers D1 and D2

\begin{tabular}{|c|c|c|}
\cline { 2 - 3 } \multicolumn{1}{c|}{} & D2 on & D2 off \\
\hline D1 on & $\left|V_{R 8}\right| \geq V_{C 1}+V_{D 1}+V_{R 11}$ & $\left|V_{R 8}\right| \geq V_{C 1}+V_{D 1}+V_{R 11}$ \\
& $\left|V_{R 9}\right| \geq V_{C 2}+V_{D 2}+V_{R 12}$ & $\left|V_{R 9}\right|<V_{C 2}+V_{D 2}+V_{R 12}$ \\
\hline D1 off & $\left|V_{R 8}\right|<V_{C 1}+V_{D 1}+V_{R 11}$ & $\left|V_{R 8}\right|<V_{C 1}+V_{D 1}+V_{R 11}$ \\
& $\left|V_{R 9}\right| \geq V_{C 2}+V_{D 2}+V_{R 12}$ & $\left|V_{R 9}\right|<V_{C 2}+V_{D 2}+V_{R 12}$ \\
\hline \multicolumn{2}{|c}{ Source: (Richter, 2018) }
\end{tabular}

(c) EY-NC-ND 2019, Editorial Universitat Politècnica de València 


\subsection{Simplified internal model description}

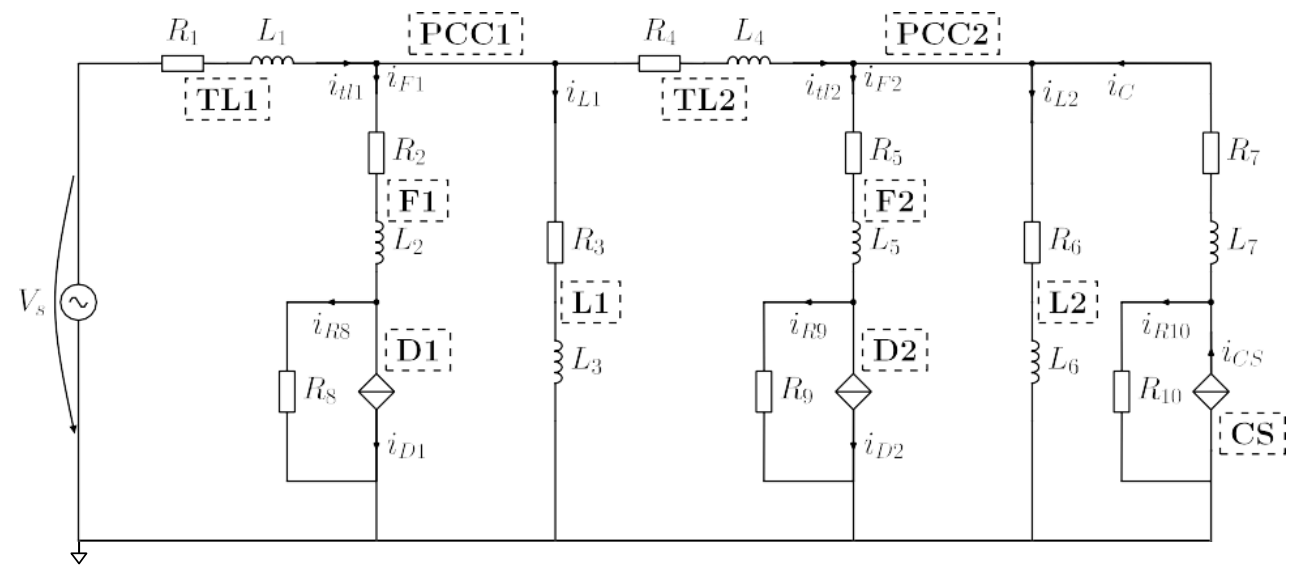

Figure 2. Simplified controller model ideal current source disturbances (Richter, 2018).

For the controller, the simpler model in Figure 2 is used to reduce complexity for the MPC. The difference is the replacement of rectifiers by ideal current sources. By excluding the switching from the rectifiers the four mode model is disregarded, leading to a single discrete-time state space model with signal vectors

$$
\begin{aligned}
\mathbf{x} & =\left[\begin{array}{lllllll}
i_{t l 1} & i_{t l 2} & i_{L 1} & i_{L 2} & i_{F 1} & i_{F 2} & i_{C}
\end{array}\right]^{\mathrm{T}}, \\
u & =i_{C S}, \\
\mathbf{d} & =\left[\begin{array}{llll}
V_{S} & i_{D 1} & i_{D 2}
\end{array}\right]^{\mathrm{T}},
\end{aligned}
$$

and corresponding matrices $\mathbf{A} \in \mathbb{R}^{7 \times 7}, \mathbf{B} \in \mathbb{R}^{7 \times 1}$, and $\mathbf{E} \in \mathbb{R}^{7 \times 3}$.

This makes a mismatch between the plant model as in (2) and the controller model.

However, the MPC feedback from the plant corrects the states mismatch in each iteration.

\section{Shape class model predictive control}

This section introduces the harmonic shape class control concept, while describing the necessary steps to reformulate a standard quadratic MPC into an $\mathrm{LS}^{3} \mathrm{MPC}$.

\subsection{Harmonic shape class}

The harmonic shape class defines the reference dynamics of the $\mathrm{LS}^{3} \mathrm{MPC}$ to compensate the total harmonic distortion (THD). The dynamics are given by the solution of the initial value problem of the homogeneous ordinary second order differential equation (ODE)

$$
\frac{\mathrm{d}^{2} x(t)}{\mathrm{d} t^{2}}+(2 \pi f)^{2} x(t)=0,
$$


that leads to a fundamental harmonic signal $x(t)$ of frequency $f$, zero offset, and arbitrary amplitude. Approximating the second derivative in (4) by forward numerical differentiation

$$
\frac{\mathrm{d}^{2} x(t)}{\mathrm{d} t^{2}} \approx \frac{2 x(k)-5 x(k+1)+4 x(k+2)-x(k+3)}{t_{s}^{2}},
$$

leads to a kernel form with harmonic shape class vector (Cateriano Yáñez et al., 2018)

$$
\mathbf{v}=\frac{1}{t_{s}^{2}}\left(\begin{array}{llll}
2+\left(2 \pi f t_{s}\right)^{2} & 5 & 4 & -1
\end{array}\right) \in \mathbb{R}^{1 \times 4} .
$$

\subsection{Linear state signal shaping model predictive control}

The unconstrained quadratic MPC minimization problem is (Maciejowski, 2002)

$$
\min _{\Delta \mathbf{U}(k)}\|\mathbf{X}(k)-\Xi(k)\|_{\mathbf{Q}}^{2}+\|\Delta \mathbf{U}(k)\|_{\mathbf{R}}^{2},
$$

with future states prediction $\mathbf{X}(k) \in \mathbb{R}^{H_{p} n}$, future states reference $\Xi(k) \in \mathbb{R}^{H_{p} n}$, control input change $\Delta \mathbf{U}(k) \in \mathbb{R}^{\left(H_{u}+1\right) m}$, cost of control error matrix $\mathbf{Q} \in \mathbb{R}^{H_{p} n \times H_{p} n}$, cost of control effort matrix $\mathbf{R} \in \mathbb{R}^{\left(H_{u}+1\right) m \times\left(H_{u}+1\right) m}$, for the prediction horizon $H_{p} \in \mathbb{N}$, and control horizon $H_{u} \in \mathbb{N}$. This quadratic optimization problem can be reformulated into the minimization of the harmonic shape class error by setting $\Xi(k)$ to zero and embedding the harmonic shape class from (6) into the cost of control error matrix $\mathbf{Q}$ (Cateriano Yáñez et al., 2018).

\section{Multiobjective optimization}

This section describes the MOO problem, including the definition of Pareto optimality, and then presents the evolutionary algorithm spMODEx, which is used to solve it.

\subsection{Multiobjective optimization problem}

The MOO problem is formally given as

$$
\min _{\zeta} \mathbf{J}(\zeta)=\min _{\zeta}\left[J_{1}(\zeta), \ldots, J_{\check{m}}(\zeta)\right]
$$

subject to $\mathbf{g}(\zeta) \leq \mathbf{0}$ and $\mathbf{h}(\zeta)=\mathbf{0}$, with $\zeta \in \mathbf{O} \subseteq \mathbb{R}^{\check{j}}$ as the vector of decision variables from $j \in\{1, \ldots, \check{m}\}$, searching the space $\mathbf{O}$, the objective function vector as $\mathbf{J}(\zeta) \in \wedge \subseteq \mathbb{R}^{\check{m}}$, and $\mathbf{g}(\zeta)$ and $\mathbf{h}(\zeta)$ as the inequality and equality constraints, (Reynoso Meza et al., 2017). This optimization problem can be solved either by aggregating the elements of the objective vector with a specific weighting $q_{k}$

$$
\min _{\zeta} \mathbf{J}(\zeta)=\min _{\zeta} \sum_{k=1}^{\check{m}} q_{k} J_{k}(\zeta)
$$


or by first computing the Pareto optimal solutions and then choosing one of these.

\subsection{Pareto optimality}

A solution vector $\zeta^{*}$ is Pareto optimal if $\nexists \zeta \in \mathbf{O}: \zeta \leq \zeta^{*}$, where $\zeta \leq \zeta^{*}$ means that $\mathbf{J}(\zeta)$ is not worse than $\mathbf{J}\left(\zeta^{*}\right)$ in all objectives, and is better in at least one objective. In other words, the set of Pareto optimal solutions is the set of cost function vectors with the property that no other vector can be found that is having all entries smaller than one of the solutions.

\subsection{Multiobjective Differential Evolution with Spherical Pruning X}

The algorithm spMODEx employs a version of the differential evolution algorithm as an evolutionary algorithm. In addition, there is an archive to continuously update the population of the differential evolution algorithm to prevent stagnation and ensure a well spread Pareto front. The pruning algorithm applied is called spherical pruning. The general working sequence of the algorithm is depicted in Figure 3.

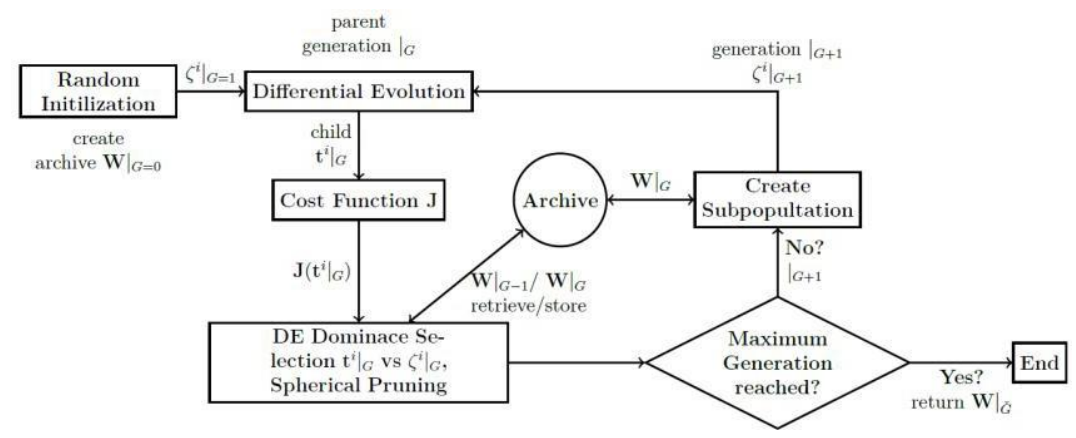

Figure 3. Graphical representation of spMODEx (Richter, 2018).

\section{Setup of experiment, simulation results and analysis}

This section describes the tuning process of an APF using a MOO algorithm. First the cost function, which is key for a successful controller tuning, and the tuning parameters are given. Then the Pareto optimal solutions are presented and discussed.

\subsection{Cost function and tuning parameters}

The cost function addresses the following six objectives: The total harmonic distortion of the currents $i_{t l}, i_{t l 2}$, and $i_{C S}$; in $J_{1}, J_{2}$, and $J_{4}$ respectively; the apparent control power $P=i_{C S} v_{C S}$ in $J_{3}$, the reactive power factor (phase shift between and $i_{C S}$ ) in $J_{5}$ and the root mean square value of $i_{t l 1}$ in $J_{6}$. For detail on the voltages and currents see Error! Reference source not found.. 
The tuning parameters are the weighting factors of the shape classes for the different states. For each state the shape class can ensure harmonic behavior by appropriately setting the entries of the weighting matrices. A degree of freedom is a factor for the shape class. These factors are chosen as decision variables.

\subsection{Simulation results}

To display the simulation results level diagrams are used. In Figure 4 the Pareto optimal solutions for the tuning of the APF are given. One solution is hereby divided into six different plots. To see one exact solution the numbers on the ordinate are used to identify a solution, i.e. the costs of all objectives for one solution are on the same level.
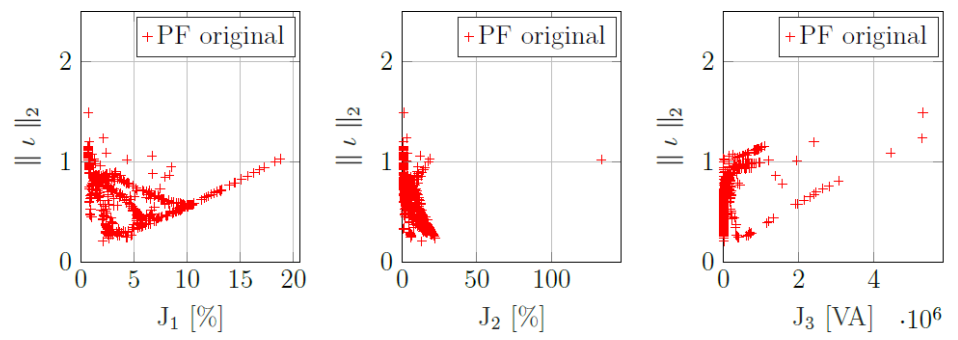

a) $J_{1}=\operatorname{THD}\left(i_{t l 1}, 10\right)$

b) $J_{2}=\mathrm{THD}\left(i_{t l 2}, 10\right)$
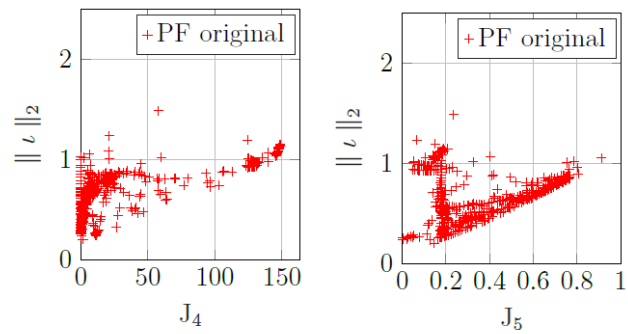

c) $J_{3}=\mathrm{ACP}(10)$

d) $J_{4}=\frac{1}{\operatorname{THD}\left(i_{C S}, 10\right)}$

e) $J_{5}=\mathrm{QPF}\left(v_{s}, i_{t l 1}, 10\right)$

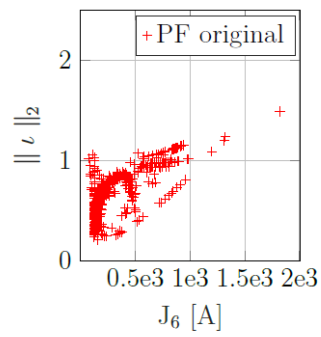

Figure 4. Level diagrams of the Pareto optimal solutions, (Richter, 2018).

\subsection{Results analysis and decision making}

To select a suitable parameters set, this solution can be further pruned. E.g. solutions having a THD value of more than 5\% are not in compliance with standard grid codes such that these solutions can be discarded. After this, a trade of between the remaining solutions can be chosen as an informed choice. 


\section{Summary and conclusions}

In this paper a controller tuning procedure for the novel $\mathrm{LS}^{3} \mathrm{MPC}$ on the basis of MOO was presented. The basics of shape classes are given and the connection to MPC is illustrated. The applied MOO algorithm spMODEx is illustrated as well. As an application example, the control of an APF is under investigation. The controller is tuned by optimizing the factors of the shape class for the different states of the given grid model.

Future research is done by adapting the optimization problem of the underlying $\mathrm{LS}^{3} \mathrm{MPC}$ from an unconstraint to a constraint optimization problem, giving additional degrees of freedom to the controller tuning, which could then efficiently be solved using the spMODEx evolutionary algorithm.

\section{Acknowledgement}

This contribution was partly developed within the project NEW 4.0 (North German Energy Transition 4.0) which is funded by the German Federal Ministry for Economic Affairs and Energy (BMWI). This paper was also partly funded by the Free and Hanseatic City of Hamburg (Hamburg City Parliament publication 20/11568).

\section{References}

Cateriano Yáñez, C., Pangalos, G., \& Lichtenberg, G. (2018). An Approach to linear state Signal Shaping by quadratic Model Predictive Control. 2018 European Control Conference (ECC), 1-6. https://doi.org/10.23919/ECC.2018.8550379

Kumar, P., \& Mishra, M. K. (2016). A comparative study of control theories for realizing APFs in distribution power systems. 2016 National Power Systems Conference (NPSC), 1-6. https://doi.org/10.1109/NPSC.2016.7858905

Liang, X. (2017). Emerging Power Quality Challenges Due to Integration of Renewable Energy Sources. IEEE Transactions on Industry Applications, 53(2), 855-866. https://doi.org/10.1109/TIA.2016.2626253

Maciejowski, J. M. (2002). Predictive Control with Constraints. Pearson education. Reynoso Meza, G., Blasco Ferragud, X., Sanchis Saez, J., \& Herrero Durá, J. M. (2017). Controller Tuning with Evolutionary Multiobjective Optimization. https://doi.org/10.1007/978-3-319-41301-3

Richter, J. (2018). Model Predictive Controller Tuning in Electrical Distribution Grids by Multiobjective Optimization. Hamburg University of Applied Sciences.

Weihe, K., Cateriano Yáñez, C., Pangalos, G., \& Lichtenberg, G. (2018, July). Comparison of Linear State Signal Shaping Model Predictive Control with Classical Concepts for Active Power Filter Design. 167-174. https://doi.org/10.5220/0006910401670174 